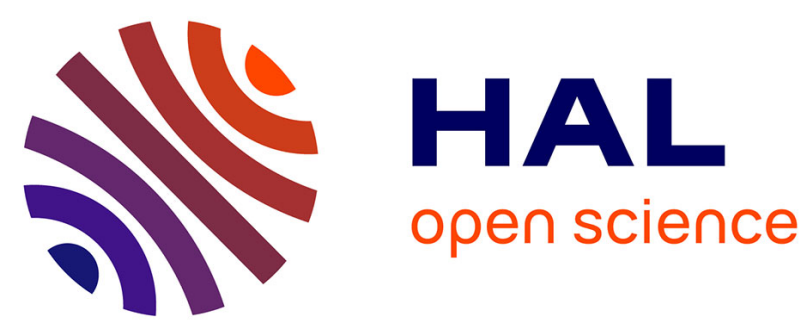

\title{
Self-similar gravity wave spectra resulting from the modulation of bound waves
}

Guillaume Michel, Benoît Semin, Annette Cazaubiel, Florence Haudin, Thomas Humbert, Simon Lepot, Félicien Bonnefoy, Michaël Berhanu, Éric

Falcon

\section{To cite this version:}

Guillaume Michel, Benoitt Semin, Annette Cazaubiel, Florence Haudin, Thomas Humbert, et al.. Selfsimilar gravity wave spectra resulting from the modulation of bound waves. Physical Review Fluids, 2018, 3 (5), pp.054801. 10.1103/PhysRevFluids.3.054801 . hal-01829955

\section{HAL Id: hal-01829955 \\ https://hal.sorbonne-universite.fr/hal-01829955}

Submitted on 4 Jul 2018

HAL is a multi-disciplinary open access archive for the deposit and dissemination of scientific research documents, whether they are published or not. The documents may come from teaching and research institutions in France or abroad, or from public or private research centers.
L'archive ouverte pluridisciplinaire HAL, est destinée au dépôt et à la diffusion de documents scientifiques de niveau recherche, publiés ou non, émanant des établissements d'enseignement et de recherche français ou étrangers, des laboratoires publics ou privés. 


\title{
Self-similar gravity wave spectra resulting from the modulation of bound waves
}

\author{
Guillaume Michel ${ }^{1}$, ${ }^{*}$ Benoît Semin ${ }^{1}$, Annette Cazaubiel ${ }^{2}$, Florence Haudin ${ }^{2}$, Thomas \\ Humbert $^{3,4}$, Simon Lepot ${ }^{4}$, Félicien Bonnefoy ${ }^{5}$, Michaël Berhanu ${ }^{2}$, and Éric Falcon ${ }^{2}$ \\ ${ }^{1}$ LPS, ENS, CNRS, Univ. Pierre et Marie Curie, Univ. Paris Diderot, Paris, France \\ ${ }^{2}$ Univ. Paris Diderot, Sorbonne Paris Cité, MSC, UMR 7057 CNRS, F-75 013 Paris, France \\ ${ }^{3}$ Present adress : Univ. du Mans, Laboratoire d'Acoustique, UMR 6613 CNRS, F-72 085 Le Mans, France \\ ${ }^{4}$ Univ. Paris-Saclay, CEA-Saclay, SPEC, DRF, \\ UMR 3680 CNRS, F-91 191 Gif-sur-Yvette, France and \\ ${ }^{5}$ École Centrale de Nantes, LHEEA, UMR 6598 CNRS, F-44 321 Nantes, France
}

(Dated: June 30, 2018)

\begin{abstract}
We experimentally study the properties of nonlinear surface gravity waves in a large scale basin. We consider two different configurations: a one-dimensional (1D) monochromatic wave forcing, and a two-dimensional (2D) forcing with bichromatic waves satisfying resonant-wave interaction conditions. For the $1 \mathrm{D}$ forcing, we find a discrete wave energy spectrum dominated at high frequencies by bound waves whose amplitudes decrease as a power law of the frequency. Bound waves (e.g. to the carrier) are harmonics superimposed on the carrier wave propagating with the same phase velocity as the one of the carrier. When a narrow frequency random modulation is applied to this carrier, the high-frequency part of the wave energy spectrum becomes continuous with the same frequency-power law. Similar results are found for the $2 \mathrm{D}$ forcing when a random modulation is also applied to both carrier waves. Our results thus show that all these nonlinear gravity wave spectra are dominated at high frequencies by the presence of bound waves, even in the configuration where resonant interactions occur. Moreover, in all these configurations, the power-law exponent of the spectrum is found to depend on the forcing amplitude with the same trend as the one found in previous gravity wave turbulence experiments. Such set of bound waves may thus explain this dependence that was previously poorly understood.
\end{abstract}

PACS numbers: 47.35.Bb, 92.10.Hm, 05.45.-a

\section{INTRODUCTION}

In the linear regime, the dynamics of the surface waves on a fluid simply consists in a set of progressive harmonic waves that verify the linear dispersion relation and do not interact with one another. To describe more complex wave fields, as the ones commonly observed in the ocean, nonlinear effects have to be taken into account. They change all of the properties mentioned above: wave trains become anharmonic, the dispersion relation now involves their amplitudes, and they may exchange energy with other wave trains.

The first two features can be found in the periodic 1D deep-water gravity wave of finite amplitude, which is no longer a sinusoidal function but is of the form

$$
\eta(x, t)=\sum_{n \geqslant 1} \eta_{n} \sin \left[n\left(\omega_{1} t-k_{1} x\right)\right], \quad \omega_{1}^{2}=g k_{1}\left(1+\epsilon^{2}+O\left(\epsilon^{4}\right)\right),
$$

where $x, t, \omega_{1}, k_{1}, \eta_{1}$ are respectively the position, the time, the angular frequency, the wavevector and the amplitude of the carrier, and $\epsilon=\eta_{1} k_{1}$ is the wave steepness. In oceanography, the term $\sin \left(\omega_{1} t-k_{1} x\right)$ of Eq. (1) is called free wave, whereas harmonics (i.e. $n \geqslant 2$ ) are called bound waves. A free wave is a wave satisfying the dispersion relation $\omega(k, \epsilon)$. A bound wave (e.g. to the carrier) is a harmonic propagating with the phase velocity of the carrier, $\left(n \omega_{1}\right) /\left(n k_{1}\right)=\omega_{1} / k_{1}$, and consequently does not verify the dispersion relation. This expression has been obtained by Stokes using an expansion in the parameter $\epsilon \equiv \eta_{1} k_{1}$, and Eq. (1) is then known as a "Stokes wave" [1]. In that case, the coefficients $\left\{\eta_{n}\right\}_{n \geqslant 2}$ of Eq. (1) and the dispersion relation have been determined analytically up to the fifth order [2] and calculations at higher orders have been numerically performed. However, these calculations have not been compared to experiments, since they characterize an unstable state: the solution (1) is subject to the modulational instability, also known as the Benjamin-Feir instability [3]. This instability of a monochromatic wave corresponds, in the spectral space, to the growth of side-bands around the carrier frequency. Similarly, the slow evolution of a wave

* guillaume.michel@ens.fr 
train can be modeled by the nonlinear Schrödinger equation [4], some exact solutions of which have been derived [5, 6] and observed in experiments (see, e.g., $[7,8]$ ).

Generally speaking, every wave field can be decomposed as a sum of free waves and bound waves, whose amplitudes and phases evolve in time. In the limit of small wave steepnesses, bound waves can be eliminated from the dynamics of the wave field, leading to the so-called Zakharov equation on which are based wave forecasting models [9]. This dynamical equation describes another feature of nonlinear surface waves: the resonant interactions between wave trains. For gravity waves, they have been identified by Phillips [10] as four-wave interactions and the associated rate of energy transfer has been computed by Hasselmann [11]. This mechanism has been experimentally studied using two intersecting wave trains (see [12] and references therein), and has been found in excellent agreement with predictions. It constitutes the building block of weak wave turbulence [13], which provides a statistical description of many interacting surface waves in the slightly nonlinear limit. The phenomenology of wave turbulence is similar to the one of hydrodynamic turbulence: energy injected at the forcing scale is transferred through local interactions and eventually dissipated by viscosity at small scales. The mean energy of the waves can then be computed between the forcing scale and the dissipative one, and wave turbulence predicts $E_{f} \propto f^{-4}$ [14], although $E_{f} \propto f^{-6}$ may also result from finite size effects [15].

Even though this direct energy cascade is observed in gravity wave turbulence experiments (see, e.g., [16-22]), it does not follow the theoretical scaling laws of weak turbulence. More precisely, the wave amplitude spectra are found self-similar and described by frequency-power laws in $f^{\alpha}$, but the exponents $\alpha \leqslant-4$ strongly depend on the forcing parameters, in contrast to the predictions of weak turbulence. These differences have been ascribed to many causes, as for instance the presence of coherent wave components [19-21], finite-size effects [23, 24] or non-local resonances [25], but the main source of discrepancies has not yet been identified. It should be noted that these high-frequency gravity spectra (i.e., at frequencies higher than the forcing one) have been studied experimentally mostly on setups close to the assumptions of wave turbulence: waves are generated in a tank with high reflection coefficient at the walls [17-21]. However, contrary to the predictions of wave turbulence according to which bound waves should be negligible (the associated correction being of the order of the wave steepness, a small parameter), the result is a mixture of free and bound waves, as evidenced by the fact that energy does not gather on the free wave dispersion relation [19, 20]. This suggests that bound waves associated to some strongly nonlinear structures could be the origin of this discrepancy.

In this article, we experimentally demonstrate that the anharmonicity of a few wave trains of large amplitude is sufficient to reproduce these self-similar spectra whose exponent depends on the forcing amplitude. To do so, we experimentally approach this problem differently: instead of considering a large number of free waves, each of them leading to bound waves, we only focus on a few free waves and their bound waves. This is achieved by generating a periodic or a slowly modulated wave field with almost no reflection at the end of the basin. The use of a beach as an efficient damping mechanism limits the occurrence of resonant interactions potentially driven by reflected waves, and allows us to focus on bound waves. We first consider the simplest forcing, i.e., a monochromatic and mostly 1D forcing. We demonstrate that the resulting wave field consists in a few free waves and a large number of bound waves, and we characterize the measured spectrum. We then study a wave train similar to the previous one, except that the frequency is slowly modulated with time. This may model the slow evolution of the wind characteristics (amplitude and direction). This is the simplest configuration in which a continuous spectrum is obtained, and where bound waves play a key role. Then, we describe how these results are affected by the addition of another free wave satisfying the resonant conditions (hereinafter referred 2D bichromatic forcing). This approach was partially initiated in [26], that reports the observation of bound waves but does not discuss how their amplitudes scale. Finally, we discuss how our results compare to a previous experiment [27], in which power-law spectra are observed for wave trains propagating toward a beach.

\section{EXPERIMENTAL SETUP}

The experimental setup is similar to that described in [12] and takes place in a large-scale basin of dimension $50 \mathrm{~m}$ long $\times 30 \mathrm{~m}$ width $\times 5 \mathrm{~m}$ deep. One side is made of 48 independently controlled paddles and the opposite one is a beach that limits wave reflection. The linear wavemaker theory is used to compute the wavemaker motion by means of classical paddle transfer function applied to the target set of water wave frequency components. The surface elevation is measured by 12 aligned resistive probes located from $x_{\min }=3.3 \mathrm{~m}$ up to $x_{\max }=29.9 \mathrm{~m}$, with $x$ the distance from the wavemaker. The positions of these probes are such that they are aligned along the daughter wave direction when resonant interactions are considered (see Sect. IV).

The prescribed wave field to the wavemaker is either 1D wave train, modulated or not (Fig. 1 left), or 2D bichromatic waves in resonance conditions (Fig. 1 right). Note that $2 \mathrm{D}$ waves are also emitted by the small spacing between paddles, whatever the forcing is. For the $2 \mathrm{D}$ forcing, the effect of the side-walls is taken into account by using the Dalrymple method to determine the forcing of each paddle to generate a homogeneous wave field in the basin (see e.g. 

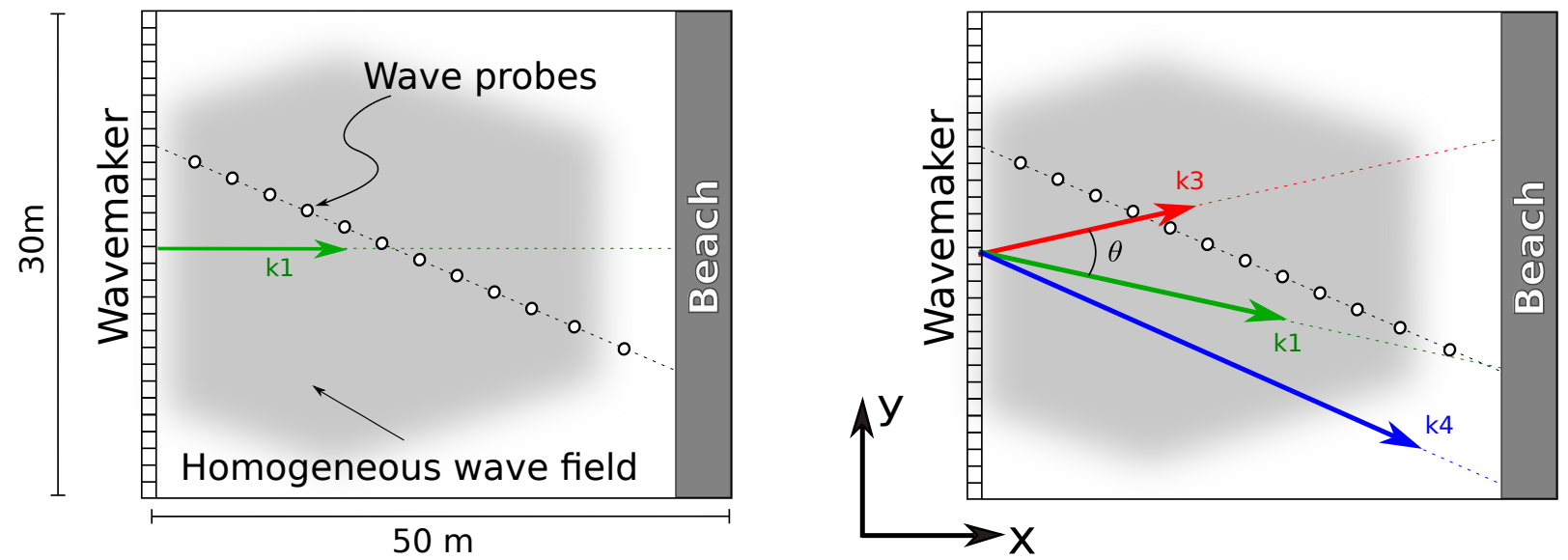

FIG. 1. Experimental setup for a 1D wave forcing (left, section II and III) and 2D bichromatic one (section IV).

[12]). The obtained wave field is quasi-uniform up to $x=25 \mathrm{~m}$ (see the gray zone of Fig. 1), and is the prescribed one. The probe at $x_{\max }$ is only used to check that there is no significant reflection at the beach, and is not included in the following analysis. We focus on a frequency range from $0.5 \mathrm{~Hz}$ to $10 \mathrm{~Hz}$, where resistive probes have a flat frequency response and water waves are in the deep-water gravity regime.

\section{1D NONLINEAR WAVE TRAIN EXPERIMENTS}

We first generate a 1D plane wave along the $x$-direction, of frequency $f_{1}=0.715 \mathrm{~Hz}$ and steepness $\epsilon=k_{1} \eta_{1}=0.10$, $\eta_{1}$ being here the prescribed value of the wave amplitude to the wavemaker. If we model the resulting wave field as a sum of progressive waves of frequencies $\left\{n \omega_{1}\right\}_{n} \geqslant 1$ and amplitudes $\left\{\eta_{n}\right\}_{n \geqslant 1}$, without prescribing the phase velocity of any of these harmonics, the wave height at a distance $x$ is of the form

$$
\eta(x, t)=\sum_{n \geqslant 1} \eta_{n} \sin \left[n \omega_{1} t-k\left(n, \omega_{1}\right) x+\phi_{n}\right]
$$

where $\phi_{n}$ is an arbitrary initial constant, and $k\left(n, \omega_{1}\right)$ is a function of $n$ and $\omega_{1} \equiv 2 \pi f_{1}$. In practice, the coefficient $\eta_{n}$ may depend slowly on $x$ as a result of, for instance, damping. Let us define $\varphi_{n}(x, t)$ as the total phase in the sine term of Eq. (2). To disentangle between free waves and bound waves, we have to compute the phase difference

$$
\Psi_{n}(x, t)=\varphi_{n}(x, t)-n \varphi_{1}(x, t)
$$
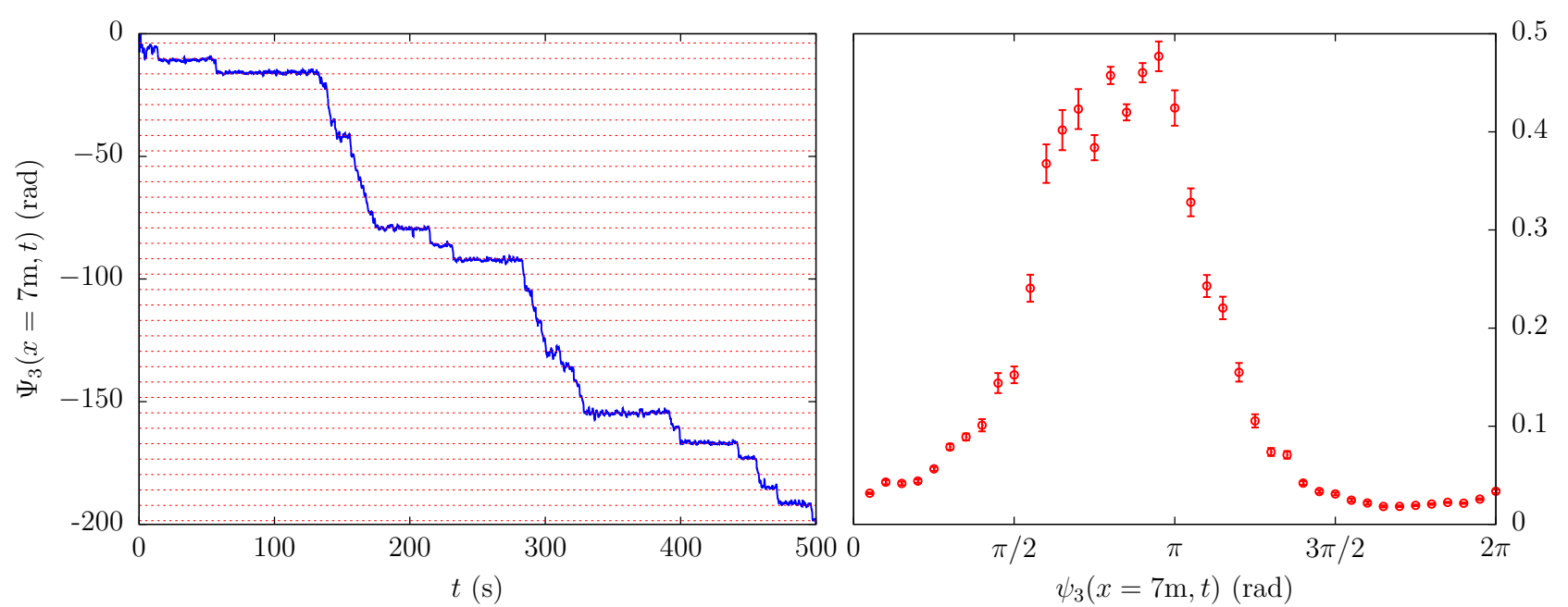

FIG. 2. Experimental phase difference $\Psi_{3}$ for the probe at $x=7 \mathrm{~m}$ as a function of time (left, where horizontal red lines are spaced by $2 \pi$ ), and its probability density function (PDF) (right). 


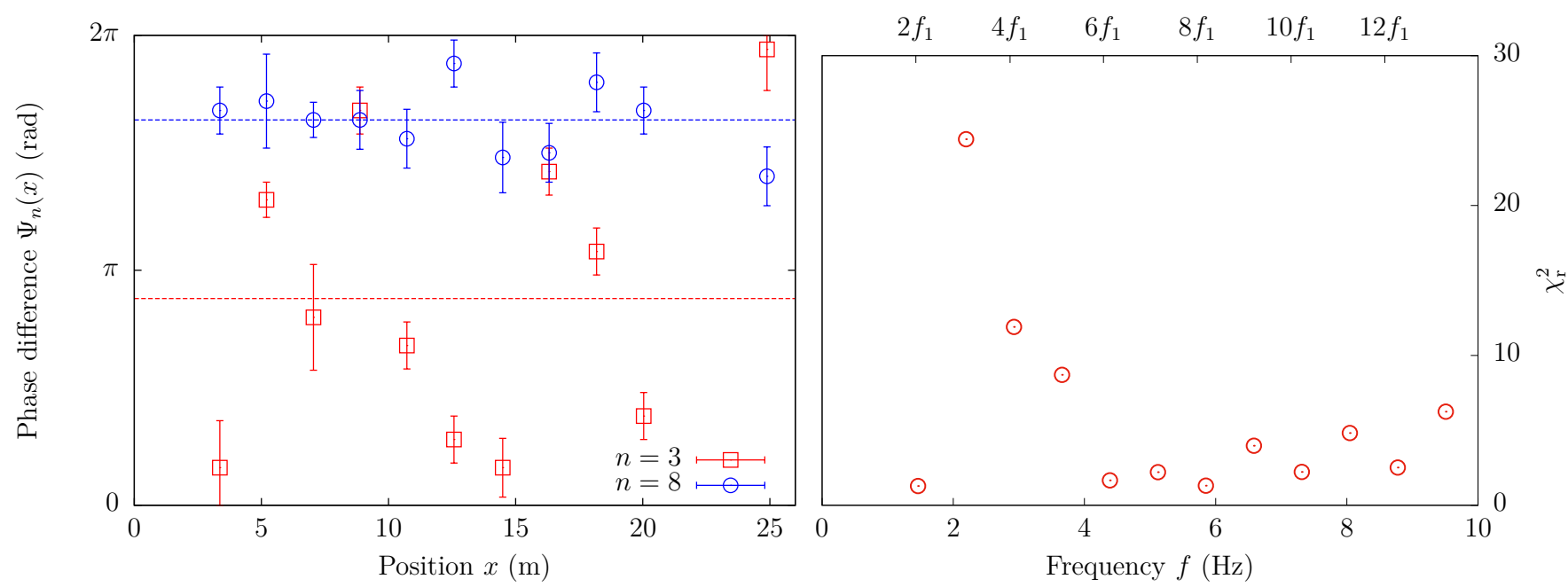

FIG. 3. Left : Evolution of the phase difference $\Psi_{n}$ with the distance $x$ to the wavemaker for $n=3$ and $n=8$. The two fits by constant functions are also reported (dashed lines). Right : coefficients $\chi_{r}^{2}$ computed for the constant fits on $\Psi_{n}(x)$.

If the $n$-th harmonic is a bound wave of the carrier wave, one has $k\left(n, \omega_{1}\right)=n k\left(\omega_{1}\right)$, and the quantity $\Psi_{n}(x, t)=\phi_{n}-\phi_{1}$ will be a constant, independent of time and space. This independence with the distance expresses the progression of a harmonic component of the wave train with the phase velocity of the carrier, $n \omega_{1} / n k\left(\omega_{1}\right)=\omega_{1} / k_{1}$ [as for the $n$-th harmonic at $n \omega_{1}$ in Eq. (1)]. However, if the $n$-th harmonic is a free wave, as well as the carrier wave, one has $k\left(n, \omega_{1}\right)=k\left(n \omega_{1}\right)$, and $\Psi_{n}(x, t)=\left[k\left(n \omega_{1}\right)-n k\left(\omega_{1}\right)\right] x+\phi_{n}-\phi_{1}$ will be independent of time, but dependent on the distance $x$. More precisely, removing initial conditions leads to $\Psi_{n}(x)-\Psi_{n}(0)=\omega_{1}^{2}\left(n^{2}-n\right) x / g$. Consequently, to discriminate between free waves and bound waves, we need to compute $\Psi_{n}(x, t)$ and look at its spatial dependence.

Experimentally, both $\eta_{n}$ and $\varphi_{n}$ can be inferred at each probe as a function of time using a Hilbert transform of the measured signal $\eta(t)$. As an example, we first compute for $n=3$, at a fixed distance $x$, the quantity $\Psi_{3}(t)$ which is experimentally found to be locked, as expected, to a fixed value $(\bmod 2 \pi)$, as evidenced in Fig. 2 left. The jumps observed in $\Psi_{3}$ correspond to very low values of $\eta_{3}$ as a result of an external perturbation, the phase no longer being properly defined. The probability density function (PDF) of $\Psi_{3}(t)$ is shown in Fig. 2 right, giving its most probable value (close to $\pi$ ) and the corresponding uncertainty (a measure of the width of the PDF, in this example close to $\pi / 4)$. Let us now compute experimentally the spatial evolution of $\Psi_{n}$ with distance $x$ for two selected harmonics as in Figure (3) left. It shows that the harmonics $n=8$ is a bound wave (since $\Psi_{8}(x)$ is roughly constant) whereas the third harmonics at $3 f_{1}$ is a free wave (since $\Psi_{3}(x)$ depends on $x$ ). For each harmonics, a fit of $\Psi_{n}[2 \pi]$ by a constant function is performed and we report in Fig.(3) right the result of the Pearson's chi-squared test $\chi_{r}^{2}$ used to measure the goodness of this fit, of order unity if the fit is accurate [28]. Eventhough the forcing is the simplest possible one, the resulting wave field is therefore a combination of free waves and bound waves: harmonics of frequencies $3 f_{1}, 4 f_{1}$ and $5 f_{1}$ are free waves, whereas high frequencies are dominated by bound waves.

We now focus on the amplitude of the different harmonics $(n \leqslant 13)$ measured by the same Hilbert transform as above. We show in Fig. 4 that they are roughly described by a frequency-power law in the range [3 Hz, $10 \mathrm{~Hz}]$. This is different from the spectrum of the Stokes wave, where the peaks' amplitudes are known to decay exponentially with the order of the harmonic [2]. This confirms that the set of bound waves observed here is not the one associated with a single free wave at $f_{1}$, but a more complex one resulting from the several free waves of low frequencies evidenced above. These free waves propagate during typically several hundred periods until they reach the beach, and may be associated to nonlinear interactions that could be crucial to sustain the anharmonic wavetrain studied here. Note that in the presence of several free waves, the bound waves do not simply consist in the superposition of each individual set of bound waves, but also contain coupling terms.

Our discrete experimental spectra are found to scale as $f^{-\alpha}$, with $-6<\alpha<-4$, and are thus compatible with the ones based on the propagation of a singular structure, $f^{-4}$ corresponding to the propagation of a one-dimensional slope break with a preserved shape $[19,29,30]$. However, there exist also structures, i.e., sets of bound waves, that are non-singular and lead to a power-law spectrum, and we thus have no proof that such singularity is observed in our experiment. The results reported in Fig. 6 (blue square) also show that the exponents $\alpha$ slightly evolve with the distance to the wavemaker. Power laws similar to the one observed here can be found in the early literature on nonlinear wave trains [31, 32], but have not been further discussed. 


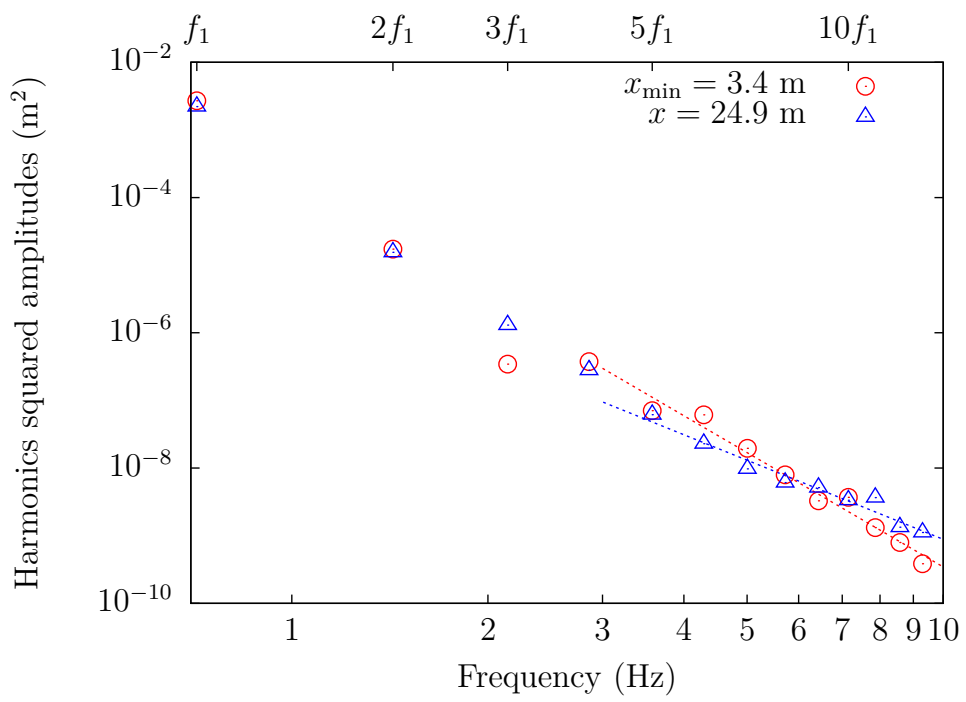

FIG. 4. Experimental evolution of the harmonic amplitudes, for $n=1$ to 13 and for two distances from the wavemaker $x$. The forcing is $1 \mathrm{D}$ and monochromatic $\left(f_{1}=0.715 \mathrm{~Hz}\right)$. Dashed lines are power laws of the form $f^{-5.6}$ and $f^{-3.9}$.

\section{EFFECT OF A NARROW FREQUENCY RANDOM MODULATION ON A 1D WAVE TRAIN}

The previous spectra are discrete, and thus correspond neither to a realistic state of the ocean, nor to the ones reported in experiments of wave turbulence. However, we will show below that continuous spectra can be obtained from discrete ones by slowly modulating the forcing frequency $f_{1}$. This will demonstrate that continuous power-law spectra dominated by bound waves exist, that strongly contrast with the ones of wave turbulence, ascribed to free waves. To illustrate the mechanism in play, we consider the following synthetic signal

$$
s(t)=\sum_{n \geqslant 1} s_{n} \cos \left\{2 \pi n\left[f_{1}+\delta f(t)\right] t\right\},
$$

where $s_{n}$ are the harmonic amplitudes. $\delta f(t)$ is a Gaussian noise of zero mean and standard deviation $\Delta f$, and is lowfiltered in time (cut-off frequency $f_{\mathrm{c}}$ ). It models a free wave at $f_{1}$ and its bound waves that are randomly modulated in frequency. To be in line with our experimental setup, we compute $s(t)$ over 500 seconds, with $f_{1}=0.715 \mathrm{~Hz}$, $\Delta f=\{0 \mathrm{~Hz}, 0.01 \mathrm{~Hz}, 0.10 \mathrm{~Hz}\}, f_{c}=f_{1} / 100$ and we arbitrary choose power-law distributed harmonic amplitude of the form $s_{n}=n^{-2}$. The associated energy spectrum $s_{n}^{2}$ is reported in Fig. 5 left.
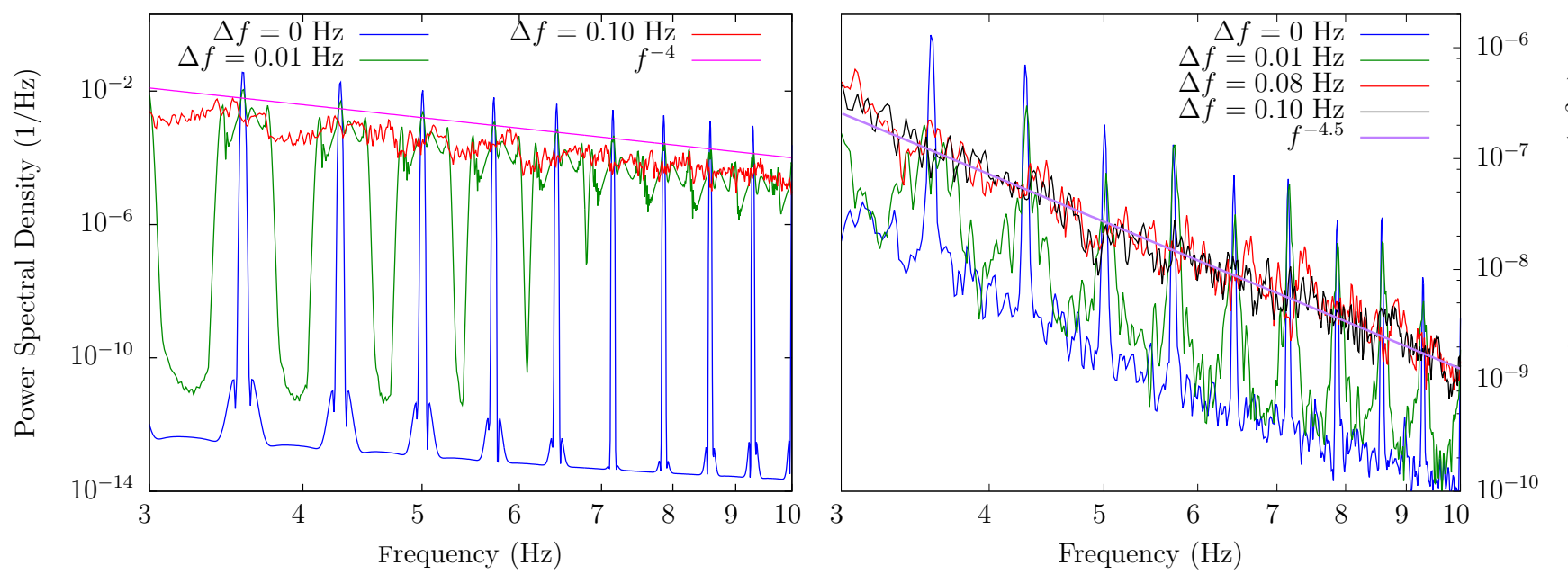

FIG. 5. Energy spectrum of a synthetic signal (left) and of experimental signals (right, $\epsilon=0.10, x=14.5 \mathrm{~m}$ ) in the case of a 1D quasi-monochromatic forcing at $f_{1}=0.715 \mathrm{~Hz}$.

Since the harmonic component of frequency $f_{n}=n f_{1}$ is modulated by a noise of standard deviation $n \Delta f$, the spectrum turns continuous when the peak corresponding to the $n+1$ th harmonics overlaps the one of the $n$ th, that 
is for a value of $n$ such that $2 n \Delta f \sim\left(f_{n+1}-f_{n}\right) \sim f_{1}$ [33]. For our range of $\Delta f$, the transition from a discrete to a continuous spectrum corresponds to crossover frequencies of $2.5 \mathrm{~Hz}(\Delta f=0.10 \mathrm{~Hz})$ and $25 \mathrm{~Hz}(\Delta f=0.01 \mathrm{~Hz})$, in agreement with the synthetic signal spectra of Fig. 5 left. Above this crossover, the continuous energy spectrum follows the same power law as the peak amplitudes of the harmonics, chosen to be $f^{-4}$ in this example.

The same peak broadening occurs in experiments when the harmonic forcing is replaced by random noise filtered at $f_{1}$ with a bandwidth $\Delta f$. Four spectra obtained over 500 seconds, for $f_{1}=0.715 \mathrm{~Hz}, \epsilon=0.10$ and $\Delta f=$ $\{0 \mathrm{~Hz}, 0.01 \mathrm{~Hz}, 0.08 \mathrm{~Hz}, 0.10 \mathrm{~Hz}\}$ are reported in Fig. 5 right. In the frequency range considered here $(3 \mathrm{to} 10 \mathrm{~Hz}), \mathrm{a}$ continuous frequency power law $f^{\alpha}$ accurately describes the spectra for high-enough frequency modulation, similarly to the synthetic signal discussed above. Once this continuous power law is developed, its value does not depend on the value of the bandwidth $\Delta f$ up to the maximal value of $0.10 \mathrm{~Hz}$ considered here. Thus, the main effect of this noise is to broaden the harmonics in the spectra up to a continuous one. The exponent $\alpha$ of this frequency-power law is therefore a characteristic of the 1D nonlinear wave, and is measured as a function of the distance for several steepnesses, when a power law fit is satisfactory. We show in Fig. 6 that the exponents resulting from a harmonic forcing (Sect. II) or from a 1D slowly modulated one (Sect. III) are roughly similar. Some results will be found in Sect. IV for a 2D resonant-wave interaction experiments (see below). This thus confirms the adequacy of the synthetic signal to model these experiments. We also observe that, as for models based on singular structures, all these spectra are steeper than $E_{f} \propto f^{-4}$.

We emphasize that the evolution of the spectra observed here and caused by a slow frequency modulation of the forcing is related to an average process over several forcing frequencies, as captured by the synthetic signal (5), and does not result from the modulation instability (or other similar processes described by the Nonlinear Schrödinger Equation, see e.g. [4]). Indeed, given the growth rate of the most unstable mode computed by Benjamin and Feir [3], $\sigma_{\text {max }}=$ $\pi \epsilon^{2} f_{1}$, and the group speed of deep-water gravity wave $g /(4 \pi f)$, the amplitude of the most unstable perturbation during its propagation over a distance $\Delta x$ grows at most by a factor $\exp \left(\sigma_{\max } \tau\right)$, with $\sigma_{\max } \tau=\left(2 \pi f_{1} \epsilon\right)^{2} \Delta x / g$. With $f_{1}=0.715 \mathrm{~Hz}, \epsilon=0.1$ and $\Delta x=25 \mathrm{~m}$ (the distance between the wavemaker and the furthest probe we use for data analysis), we obtain $\exp \left(\sigma_{\max } \tau\right) \simeq 2$. To evidence the Benjamin-Feir instability, this quantity should be increased by either tuning the characteristics of the wave field (increasing the frequency $f_{1}$ or the steepness $\epsilon$ ) or using a longer basin. For instance, the experimental study of Melville [32] has a similar $\Delta x$, but $f_{1}$ and $\epsilon$ are approximately set three times larger $\left(f_{1}=2 \mathrm{~Hz}, \epsilon \simeq 0.25\right)$, increasing $\sigma_{\max }$ by a factor $\sim 50$.

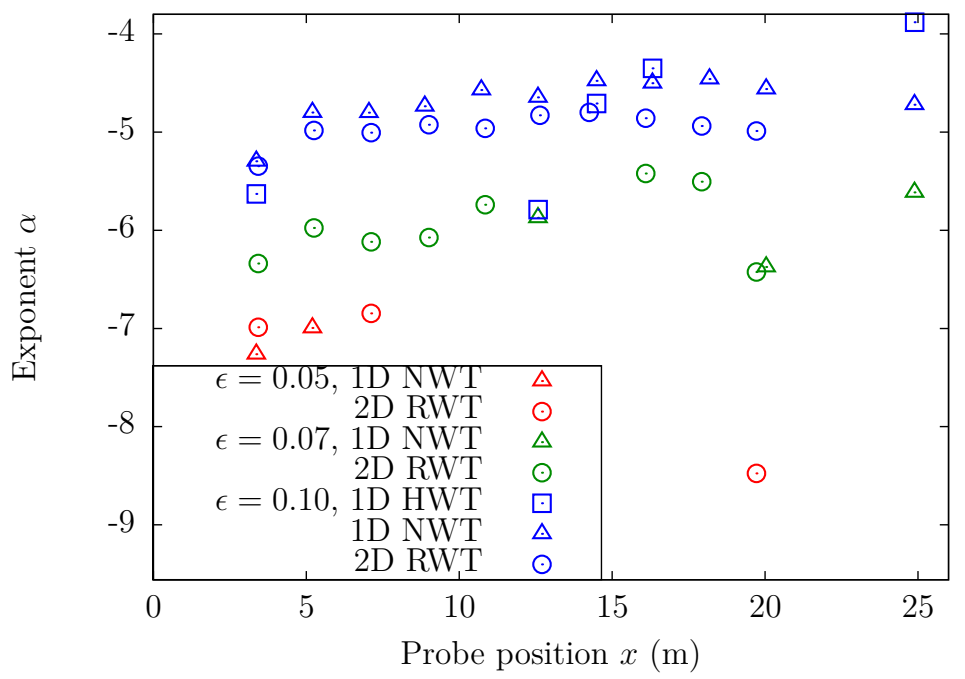

FIG. 6. Exponent $\alpha$ of the energy spectrum in $f^{\alpha}$ as a function of the distance from the wavemaker, for three steepnesses $\epsilon=\{0.05,0.07,0.10\}$. ( $\square)$ refer to a harmonic forcing (HWT, described in section II), $(\triangle)$ to a noisy wave train regardless of $\Delta f$ (NWT, described in section III) and $(\bigcirc)$ to two wave trains in resonant interaction (RWT, described in section IV)

\section{2D RESONANT-WAVE INTERACTION EXPERIMENTS}

We now present experimental results for a 2D configuration in which an important four-wave resonant energy transfer occurs at large scales. We will notably discuss the corresponding wave energy spectrum. To do so, the wavemaker is set to generate two monochromatic wave trains of frequency $f_{3}=0.715 \mathrm{~Hz}$ and $f_{1}=0.9 \mathrm{~Hz}$, called mother waves, intersecting with an angle $\theta=25^{\circ}$, as shown in Fig. 1 (right). This configuration is choosen to 
correspond to a four-wave resonance condition. It leads to the growth of a free daughter wave of frequency $f_{4}$, and wave vector $\mathbf{k}_{4}$, such that

$$
f_{4}=2 f_{1}-f_{3}, \quad \mathbf{k}_{4}=2 \mathbf{k}_{1}-\mathbf{k}_{3} .
$$

We recently investigated this resonance with a similar experimental configuration and set of parameters [12]. The characteristics of this daughter wave for the first stages of interaction (short times) are [34]:

$$
\epsilon_{4}=G \epsilon_{1}^{2} \epsilon_{3} k_{4} d, \quad \Phi=-\frac{\pi}{2}[2 \pi]
$$

where $d$ is the distance along with $\mathbf{k}_{4}(d=0$ at the wavemaker $), \epsilon_{\{1,3,4\}}$ are the steepnesses of the waves, $\Phi(t)=$ $\phi_{4}(t)+\phi_{3}(t)-2 \phi_{1}(t)$ is a combination of their instantaneous phases that are phase locked, and $G=1.32$ is the prefactor for the chosen angle.

For the present $2 \mathrm{D}$ experiments, the mother waves are changed from monochromatic at $f_{1}$ and $f_{3}$ to two filtered noises around $f_{1}$ and $f_{3}$ of similar bandwidth $\Delta f$, i.e. of wave trains with amplitudes and phases slowly varying with time compared to the growth rate of the daughter wave. Compared to the 1D slowly modulated wave train of Sect. III, the only change is that we now introduce resonant interactions between the mother waves. For simplicity, we choose mother waves of same mean steepness: $\left\langle\epsilon_{1}\right\rangle=\left\langle\epsilon_{3}\right\rangle \equiv \epsilon$, where $\langle\cdot\rangle$ stands for a time average. For the recorded wave elevation at one probe, we infer the instantaneous amplitude and phase of the mother waves, and of the daughter wave, using a Hilbert transform (see [12]). In Fig. 7 (left), we show that the daughter wave amplitude is in agreement with Eq. (6) even if the mother waves are not purely monochromatic. The phase locking is also observed in Fig. 7 (right) when the amplitude of the daughter wave is large enough, so that $\Phi$ can be accurately measured. This demonstrates that the free daughter wave is a quasi-static response to the slow forcing evolution.
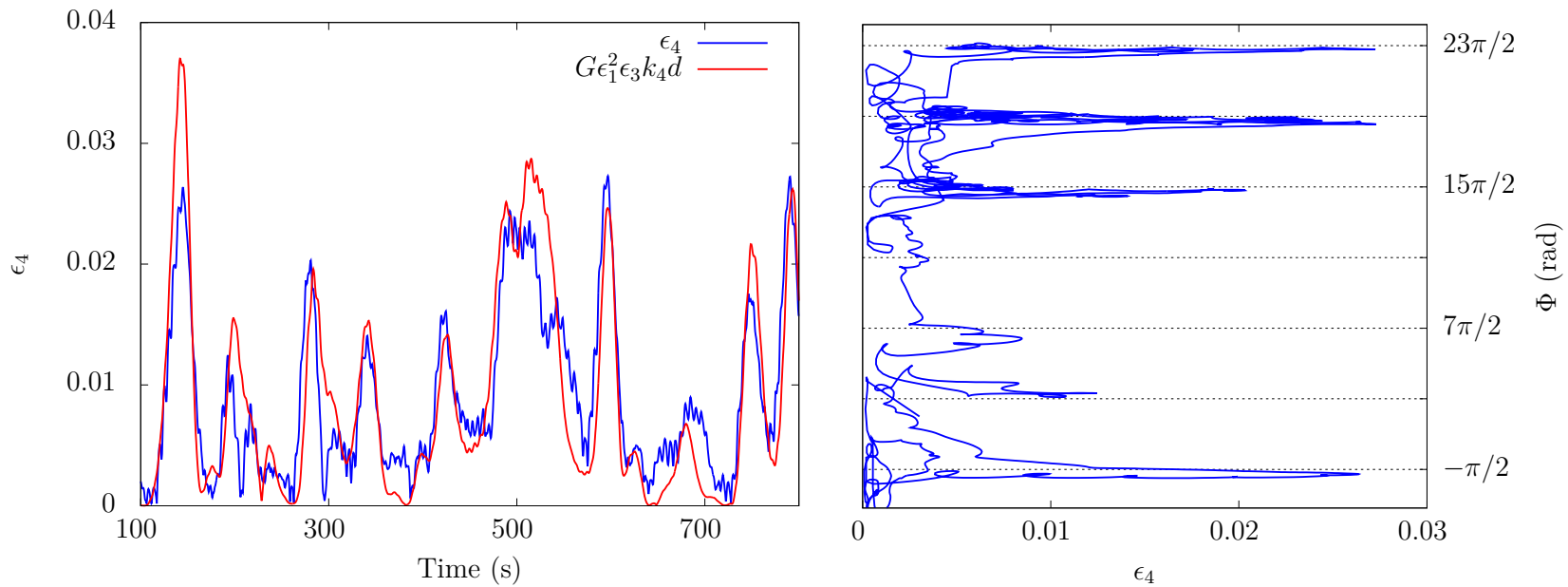

FIG. 7. Left : Temporal evolution of the daughter wave steepness $\epsilon_{4}$ experimentally measured (blue) and the theoretical prediction (red), with no fitting parameter. Right : total phase $\Phi$ vs $\epsilon_{4}$, showing the phase-locking $\Phi=-\frac{\pi}{2}[2 \pi]$. These data are for $x=19.8 \mathrm{~m}, \Delta f=0.02 \mathrm{~Hz}$ and $\epsilon=0.05$

We now consider the high-frequency part of the energy-wave spectrum (from $3 \mathrm{~Hz}$ to $10 \mathrm{~Hz}$ ), as reported in Fig. 8 , for different frequency bandwidths $\Delta f$. For $\Delta f=0 \mathrm{~Hz}$, it reduces to a set of peaks of the form $n f_{1}+m f_{3}$, with $n$ and $m$ integer. This corresponds to a mixture of free waves and bound waves, that can hardly be distinguished without spatial measurement. Free waves result from resonances in cascade that come from four-wave interactions. At high enough $\epsilon$, cascade of resonances involves the interaction between primary daughter wave $\left(f_{4}\right)$ and a mother wave $\left(f_{1}\right.$ or $f_{3}$ ), leading to the generation of a secondary daughter wave (saying $f_{5}$ ) that may also interact with a mother wave, and so on. This corresponds to the building of the direct energy cascade in the framework of wave turbulence. The first steps of this cascade can be investigated with this setup and will not be described here [35]. Here, we focus on the effect of a narrow frequency noise. Similar to Sect. III, the addition of a noise makes continuous the high-frequency part of the spectrum. It is also well-described by a power law independent of $\Delta f$ in the range considered here, as shown in Fig. 8. The minimal value of $\Delta f$ for which the spectrum is continuous is smaller than in the case of a $1 \mathrm{D}$ wave train (see Fig. 5 right) because the interaction of two waves generates more peaks in the spectrum.

Finally, we report in Fig. 6 the exponent of the power law describing these high-frequencies. They are similar to the ones describing the spectra of Sect. III corresponding to the $1 \mathrm{D}$ nonlinear wave. We therefore propose that, even though four-wave resonant interactions occur and efficiently describe some part of the spectrum (as the amplitude and phase at $f_{4}$ ), the high-frequency part of the spectrum is still dominated by bound waves. 


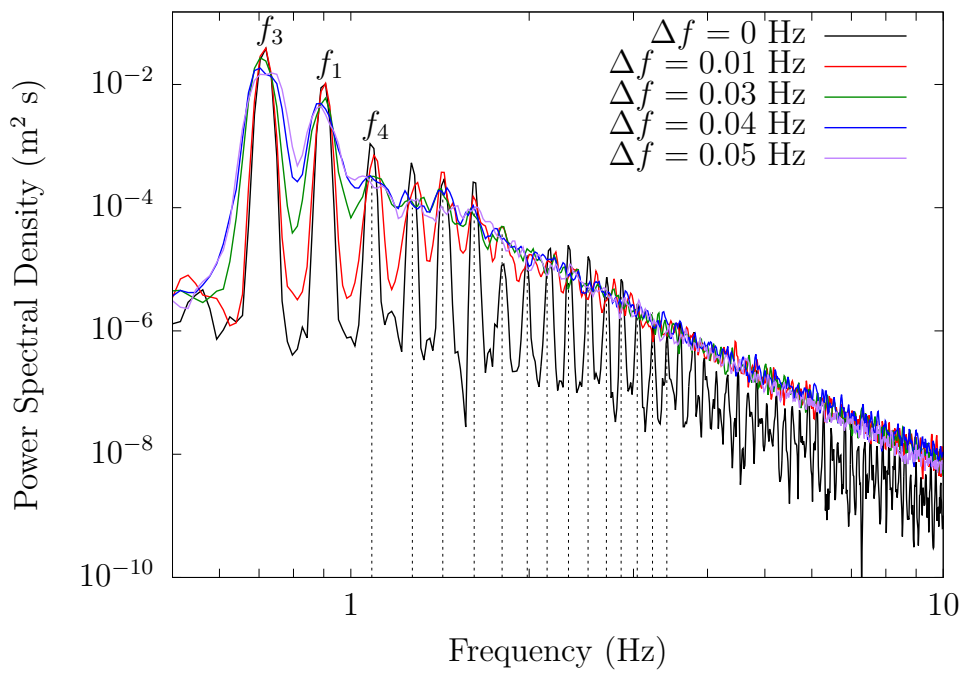

FIG. 8. Wave energy for a $2 \mathrm{D}$ bichromatic forcing (at $f_{1}$ and $f_{3}$ ), for different frequency modulation or bandwidth $\Delta f$. These data correspond to $x=10.85 \mathrm{~m}$ and $\epsilon=0.10$. For $\Delta f=0, f_{4}$ corresponds to the primary daughter wave, and dashed lines to the other free or bound waves, of frequencies of the form $n f_{1}+m f_{3}$ with $n$ and $m$ integers (up to $3.415 \mathrm{~Hz}$ for clarity).

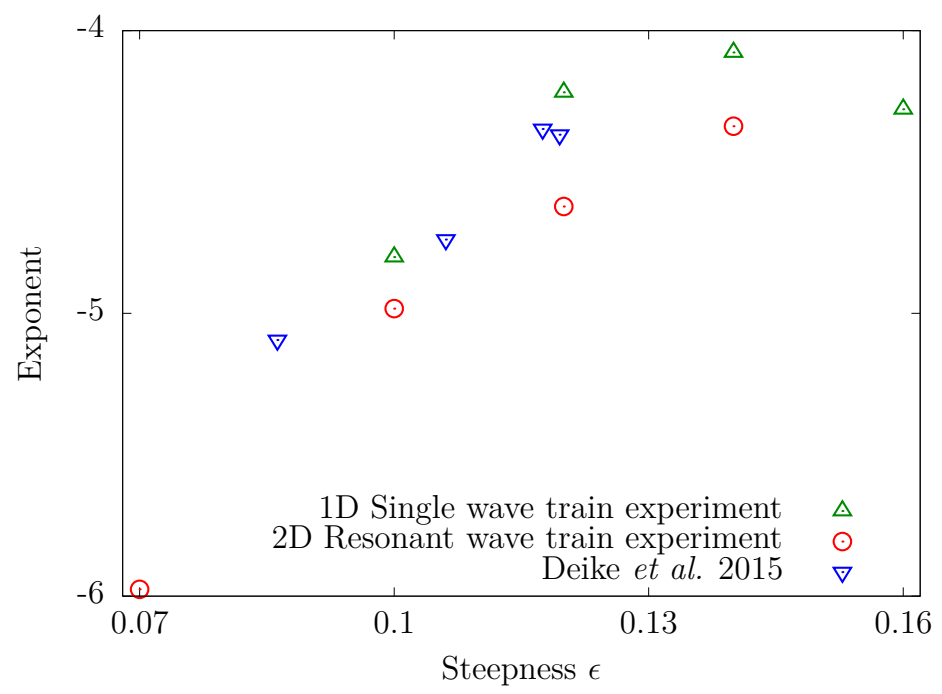

FIG. 9. Exponent of the frequency-power law spectrum of the wave energy as a function of the wave steepness $\epsilon$ for the 1D single noisy wave train experiment (1D NWT Sect. III), the 2D resonant wave train experiment (2D RWT Sect. IV), and the experiments of Deike et al. [27] in the configuration with a beach. These data correspond to $x=7.05 \mathrm{~m}(1 \mathrm{D}$ NWT) and $x=7.4 \mathrm{~m}(2 \mathrm{D}$ RWT and [27]).

\section{COMPARISON WITH PREVIOUS EXPERIMENTS}

The results of the present work can be compared to the previous experiments of Deike et al. [27], in which a wavemaker generates a 1D random wave field in a smaller basin. Two boundary conditions are considered by the authors, a beach and a reflecting vertical wall. This corresponds to two very different regimes: resonant interactions are unlikely to be efficient if a beach limits reflection, and conversely resonances in cascade are expected in presence of a wall. However, these two regimes share a similar frequency signature: both energy spectra are frequency-power laws of exponents between -7 and -4 , but the variations with the steepness are different [27]. We report in Fig. 9 the exponents obtained in [27] with a beach and narrow bandwidth (Jonswap) forcing, together with the ones obtained in this set of experiments at approximately the same distance. These exponents are similar, showing thus that the spectra measured with a beach can then be ascribed to bound waves, as proposed by the authors.

Finally, note that the observation of the predictions of gravity wave turbulence requires strict experimental conditions: the boundary conditions should be reflecting and the forcing should generate many waves with different 
frequencies, phases and directions instead of a single and high-amplitude wave train. This has for instance been achieved for the observation of the inverse cascade [36] or in a large tank with reflecting vertical walls [27].

\section{CONCLUSION}

A typical state of the ocean consists of a large number of gravity waves interacting one with another. The statistics of such a wave field can be derived in the framework of weak turbulence, in the limit of weak nonlinearity, no dissipation, and infinite size system. Unless care is taken of to stay in this narrow range of parameters, in situ measurements as well as laboratory experiments reveal deviations from these predictions, independently of the basin size (0.5 $\mathrm{m}$ to 50 m) $[13,16,17,21,27,37,38]$. In particular, the exponent of the frequency-power law energy spectrum depends on the forcing amplitude, and bound waves are observed. Here, in contrast to typical experiments of wave turbulence in which reflecting walls are used, and many waves of different frequencies and directions are generated, we report the frequency-spectra associated to only a few strongly nonlinear wave trains propagating toward a beach.

We have characterized the harmonics of a $1 \mathrm{D}$ gravity wave forced at a single frequency, which are essentially bound waves at high frequencies. The energy of these harmonics has a discrete spectrum that is described by a frequencypower law. For a 1D quasi-monochromatic wave train, i.e., a slowly modulated wave, the energy spectrum becomes continuous at high frequencies and is well fitted by a power law. The exponent of this power law is the same as the one obtained for a monochromatic forcing, showing that this frequency-power law exponent is also likely to originate from bound waves. To investigate whether the presence of resonant interactions and of several free waves affects these statistics (i.e., if the high-frequency part of the spectra remains or not dominated by bound waves), we then forced two resonant mother waves in a $2 \mathrm{D}$ configuration. We have shown that this regime involves both free waves and bound waves: the daughter wave as well as some harmonics are free waves and their amplitudes can be predicted from resonant interaction theory. We report continuous power law spectra of exponents close to the ones of the single wave train experiment, indicating that high frequencies are still likely to be dominated by bound waves. Note that to distinguish between free waves and bound waves, a spatial measurement should be performed by means for instance of many probes or optical measurement of wave height on the whole surface.

The main breakthrough of our study is that we are able to reproduce the usual experimental deviations from gravity wave turbulence predictions with a single 1D gravity wave train subjected to a narrow frequency random modulation. More precisely, the high-frequency part of the spectrum is a power law whose exponent depends on the forcing parameters, as observed in ocean [39] and in well-controlled laboratory experiments [16-21, 38]. The presence of such anharmonic wave trains in these experiments would therefore explain both the observation of bound waves and the high-frequency energy spectrum. One reason may be that in some laboratory experiments, the wave field is dominated by the wave trains of large amplitude directly emitted by the wavemakers. However, this has been proven not to be always the case: for instance, the experiments of Deike et al. with a fixed wall instead of a beach describe a regime of wave turbulence in which the $1 \mathrm{D}$ nature of the forcing is lost, while spectra steeper than $f^{-4}$ are observed [27]. Another possibility is that the anharmonic wave trains studied here are similar to some strongly nonlinear coherent structures that would spontaneously rise in wave turbulent systems and dominate the background turbulent free wave field. This would provide a complementary approach of turbulent states: while weak turbulence theory is suited to describe the local energy cascade taking place in the presence of many different free waves, starting from one or a couple of nonlinear wave trains could be relevant to take into account coherent structures.

\section{ACKNOWLEDGMENT}

This work has been supported by ANR Dysturb 17-CE30-0004-02 and by ANR Turbulon 12- BS04-0005.

[1] G. G. Stokes, On the Theory of Oscillatory Waves, Camb. Trans 8, 441 (1847). See also G. B. Whitham, Linear and Nonlinear Waves, John Wiley \& Sons Inc. (1974).

[2] J. D. Fenton, J. Waterway, A Fifth-order Stokes Theory for Steady Waves, Port. Coastal and Ocean Eng. Div. ASCE 111, $216(1985)$.

[3] T. B. Benjamin and J. E. Feir, The disintegration of wave trains on deep water Part 1. Theory, J. Fluid Mech. 27, 417 (1967).

[4] V. E. Zakharov, Stability of Periodic Waves of Finite Amplitude on the Surface of a deep Fluid, J. Appl. Mech. Tech. 9, 86 (1968). 
[5] V. E. Zakharov and A. B. Shabat, Exact theory of two-dimensional self-focusing and one-dimensional self-modulation of waves in nonlinear media, Sov. Phys.-JETP 34, 62 (1972).

[6] D. H. Peregrine, Water waves, nonlinear Schrödinger equations and their solutions, Austral. Math. Soc. Ser. B 25, 16 (1983).

[7] H. C. Yuen and B M. Lake, Nonlinear deep water waves: Theory and experiment, Phys. Fluids 18, 956 (1975).

[8] A. Chabchoub, N. P. Hoffmann and N. Akhmediev, Rogue Wave Observation in a Water Wave Tank, Phys. Rev. Lett. 106, 204502 (2011).

[9] J. B. Zicker, The Science of Ocean Waves (Johns Hopkins University Press, 2013).

[10] O.M. Phillips, On the dynamics of unsteady gravity waves of finite amplitude Part 1. The elementary interactions, J. Fluid Mech. 9, 193 (1960).

[11] K. Hasselmann, On the non-linear energy transfer in a gravity-wave spectrum Part 1. General theory, J. Fluid Mech. 12, 481 (1962).

[12] F. Bonnefoy, F. Haudin, G. Michel, B. Semin, T. Humbert, S. Aumaître, M. Berhanu, and E. Falcon, Observation of resonant interactions among surface gravity waves, J. Fluid Mech. 805, R3 (2016).

[13] S. Nazarenko, Wave Turbulence (2001), and V. E. Zakharov, V. S. L'vov and G. Falkovich, Kolmogorov Spectra of Turbulence 1: Wave Turbulence, Series in Nonlinear Dynamics, Springer (1992).

[14] V. E. Zakharov and N. N. Filonenko, Energy Spectrum for Stochastic Oscillations of a Fluid Surface, Sov. Phys. Dokl. 11, 881 (1967).

[15] S. V. Nazarenko, Sandpile behaviour in discrete water-wave turbulence, J. Stat. Mech., L02002 (2006).

[16] E. Falcon, C. Laroche and S. Fauve, Observation of Gravity-Capillary Wave Turbulence, Phys. Rev. Lett. 98, 094503 (2007).

[17] P. Denissenko, S. Lukaschuk, and S. Nazarenko, Gravity Wave Turbulence in a Laboratory Flume, Phys. Rev. Lett. 99, 014501 (2007).

[18] S. Lukaschuk, S. Nazarenko, S. McLelland, and P. Denissenko, Gravity Wave Turbulence in Wave Tanks: Space and Time Statistics, Phys. Rev. Lett. 103, 044501 (2009).

[19] S. Nazarenko, S. Lukaschuk, S. McLelland, and P. Denissenko, Statistics of surface gravity wave turbulence in the space and time domains, J. Fluid. Mech. 642, 395 (2010).

[20] E. Herbert, N. Mordant, and E. Falcon, Observation of the Nonlinear Dispersion Relation and Spatial Statistics of Wave Turbulence on the Surface of a Fluid, Phys. Rev. Lett. 105, 144502 (2010).

[21] P. Cobelli, A. Przadka, P. Petitjeans, C. Lagubeau, V. Pagneux, and A. Maurel, Different Regimes for Water Wave Turbulence, Phys. Rev. Lett. 107, 214503 (2011).

[22] L. Deike, M. Berhanu, and E. Falcon, Energy flux measurement from the dissipated energy in capillary wave turbulence, Phys. Rev. E 89, 023003 (2014).

[23] V. E. Zakharov, A. O. Korotkevish, A. N. Pushkariv, and A. I. Dyachenko, Mesoscopic Wave Turbulence, J. Exp. Theor. Phys. Lett. 82, 487 (2005).

[24] E. Kartashova, S. Nazarenko and O. Rudenko, Resonant interactions of nonlinear water waves in a finite basin, Phys. Rev. E 78, 016304 (2008).

[25] Q. Aubourg and N. Mordant, Nonlocal Resonances in Weak Turbulence of Gravity-Capillary Waves, Phys. Rev. Lett. 114, $144501(2015)$

[26] T. M. A. Taklo, K. Trulsen, O. Gramstad, H. E. Krogstad, and A. Jensen, Measurement of the dispersion relation for random surface gravity waves, J. Fluid Mech. 766, 326 (2015).

[27] L. Deike, B. Miquel, P. Gutiérrez, T. Jamin, B. Semin, M. Berhanu, E. Falcon, and F. Bonnefoy, Role of the basin boundary conditions in gravity wave turbulence, J. Fluid Mech. 781, 196 (2015).

[28] J. Taylor, An Introduction to Error Analysis (University Science Books 1997).

[29] E. A. Kuznestov, Turbulence spectra generated by singularities, JETP Lett. 80, 83 (2004).

[30] C. Connaughton, S. Nazarenko, and A. C. Newell, Dimensional analysis and weak turbulence, Physica D 184, 86 (2003).

[31] B. M. Lake, H. C. Yuen, H. Rungaldier, and W. E. Ferguson, Nonlinear deep-water waves: theory and experiment. Part 2. Evolution of a continuous wave train, J. Fluid Mech. 83, 49 (1977).

[32] W. K. Melville, The instability and breaking of deep-water waves, J. Fluid Mech. 115, 165 (1982).

[33] see Carson's bandwidth rule for frequency-modulated signals, J.R. Carson, Notes on the theory of modulation, Proc. IRE, 10, 57 (1922).

[34] M. S. Longuet-Higgins, Resonant interactions between two trains of gravity waves, J. Fluid Mech. 12, 321 (1962).

[35] F. Bonnefoy et al., in preparation.

[36] L. Deike, C. Laroche, and E. Falcon, Experimental study of the inverse cascade in gravity wave turbulence, Europhys. Lett. 96, 34004 (2011).

[37] Q. Aubourg and N. Mordant, Investigation of resonances in gravity-capillary wave turbulence, Phys. Rev. Fluids, 1, 023701 (2016).

[38] Q. Aubourg, A. Campagne, C. Peureux, F. Ardhuin, J. Sommeria, S. Viboud and N. Mordant, Three-wave and four-wave interactions in gravity wave turbulence, Phys. Rev. Fluids, 2, 114802 (2017)

[39] N. E. Huang, S. R. Long, C.-C. Tung, Y. Yuen and L. F. Bliven, A unified two-parameter wave spectral model for a general sea state, J. Fluid Mech. 112, 203 (1981). 